\title{
Locomotion analysis and its applications in neurological disorders detection: state-of-art review
}

\author{
Wenlong Tang $\cdot$ Di Su
}

Received: 21 June 2012/Revised: 25 September 2012/Accepted: 2 October 2012/Published online: 14 November 2012

(C) Springer-Verlag Wien 2012

\begin{abstract}
Driven by the need of non-invasive diagnosis in clinical practice, behavioral screening and tests have attracted more and more research interests recently. Locomotion analysis, as an important division of behavioral tests, has been widely used in diagnostic decision making, comparative biomechanics, biometric identification and forensics, etc. Plenty of instruments and approaches for locomotion analysis have been developed in both academic and industrial fields to detect diseases clinically, especially neurological disorders. Thus, it is crucial to summarize the methodology and applications of locomotion analysis to help researchers better understand this field. In this review, we focus on describing some recently developed locomotion instruments and their applications in detecting neurological disorders. We also discuss the recent progress of locomotion studies and their pros and cons.
\end{abstract}

Keywords Locomotion - Detection ·

Neurological disorders

\section{Introduction}

Early diagnosis of diseases is the best protection against their effects. Thus, an efficient and smart approach to detect some specific diseases early is a key to protect

\footnotetext{
W. Tang $(\bowtie)$

Department of Electrical and Computer Engineering,

The University of Alabama, 101 Houser Hall,

Tuscaloosa, AL 35487, USA

e-mail: wtang11@bama.ua.edu

D. $\mathrm{Su}$

School of Medicine, The University of Alabama

at Birmingham, Birmingham, AL, USA
}

people. What kind of approaches can be called efficient and smart? There are several criteria to follow. First of all, the detected results should be accurate. Second, the detection approach should be simple, minor or non-invasive. Third, the cost should be low. Driven by the demands of these criteria, many detecting approaches have been developed, such as specimen tests, computed tomography (CT), magnetic resonance imaging (MRI), behavioral screening and tests, etc. A particular disease may have one or more appropriate options. Locomotion analysis, a division of behavioral tests, has attracted increasing research interests (Thomsen et al. 2012; Wu et al. 2012; Reiner et al. 2012; Suresh Babu et al. 2012; Young et al. 2012), and it is potentially the best early detection solution for some particular diseases.

Locomotion analysis, also called gait analysis mostly, is a technology that aims to extract useful information by studying the motions of animal models or human subjects. Moreover, locomotion analysis matches the three criteria perfectly as an efficient, non-invasive, and economical approach. The technology has been used since the seventeenth century, and its development has accelerated recently with the advent of computer technology (for a review see Morris and Lawson 2009). The utility of locomotion analysis is broad now, and has been used in the detection of limps in dairy cows (Liu et al. 2009a), aging research (Woollacott and Shumway-Cook 2002), neurological disorders detection (Tang et al. 2012), etc. Toro et al. (2002) published a review of observational gait assessment in clinical practice, and the report focused on the approaches to perform gait analysis, such as "naked eye" observation of gait and video-based observational gait assessment. Gait assessment is an everyday responsibility for some practicing therapists. Visual assessment, which is universally used for this purpose, has been shown to be 
unreliable. Thus, measurement of the temporal and spatial factors of gait objectively has been found to be clinically meaningful (Wall and Brunt 1996). Moreover, locomotion analysis has been rapidly expanding in the past few years and its impact on the diagnosis of neurological disorders has been rapidly increased. Locomotion analysis has become an important approach for the diagnosis of many neurological disorders (Tien et al. 2010). As a result, it is necessary to review the recent progress of locomotion studies and their applications in detecting neurological disorders.

A system of locomotion analysis commonly consists of two components: an instrument which collects data and a computational model which generates reliable detection results based on the data set collected. The rest of this review article is organized as follows: an introduction to the instruments, the data generated by the instruments, and approaches used to analyze the locomotion data will be presented in Sect. 2, including laboratory prototypes and commercially available instruments, for animal models as well as human beings; Sect. 3 will describe the applications of locomotion analysis in the detection of neurological disorders and their performance. Discussion and conclusion will be delivered in Sects. 4 and 5, respectively.

\section{Instruments for locomotion analysis}

One early locomotion analysis method, which is still used today, is to dip the paws of animal models or human subjects' feet in ink and make them walk on a piece of paper (Costa et al. 1999). This method is called "foot print," as shown in Fig. 1. The subject leaves a series of footprints on a paper track. Running time, number of steps, and distance between fore and hind limbs on left and right sides are measured. With the development of computer science and technology, many locomotion technologies based on computers have been developed. The advanced locomotion analysis instruments which are used to collect locomotion signals can be classified into two major types: sensor platforms and wearable sensors.

\subsection{Sensor platforms}

Full and Tu (1990) developed a sensor platform locomotion analysis system as shown in Fig. 2. They measured vertical and horizontal ground reaction forces (GRFs) using a miniature force platform based on a design by Heglund (1981). A platform measuring $10.7 \mathrm{~cm} \times 6 \mathrm{~cm} \times 0.06 \mathrm{~cm}$ and weighing $3.07 \mathrm{~g}$ was constructed of model aircraft plywood and mounted on four brass beams. The locomotion analysis for insects (Full and Tu 1990) and rats (Muir and Whishaw 1999a, b) were performed on the system (Fig. 2). A system with a bigger size was built for rats with $3 \mathrm{~m}$ in

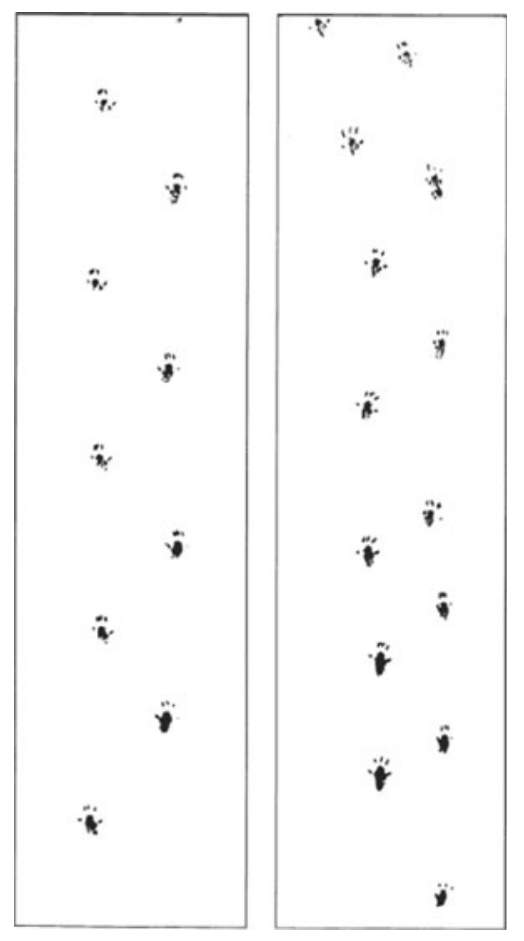

Fig. 1 Black waterproof ink was applied to the hind paws of individual mice. The animals were then placed at one end of a long and narrow tunnel $\left(10 \times 10 \times 70 \mathrm{~cm}^{3}\right)$, which they spontaneously enter and partially or totally transverse. A clean sheet of white paper (35.5-cm long) was placed on the floor of the tunnel to record the paw prints (adapted from Costa et al. 1999)

length and $20 \mathrm{~cm}$ in width. The GRFs were analyzed, but no models were constructed based on the results of the studies.

Another locomotion analysis system was developed by Clarke and Still (2001) for laboratory mice models. The platform was made of transparent glass. Two cameras under the platform captured the video of the walking mice. In addition, two force load cells measured GRFs in the vertical direction and supported the platform. The locomotion parameters that were measured by the system were velocity, stride time, stance time, swing time, and maximum GRFs in the vertical direction.

Tang et al. (2009b) developed a gait analysis system for laboratory rat models as shown in Fig. 3. The system had an entrance, a sensor module, and an exit. The dimensions of the entrance and exit floor-plates can be found in Tasch et al. (2008). The sensor module consisted of two side-byside transparent Plexiglas floor-plates. Each floor plate was suspended by four load cells. These load cells measured the vertical GRF components. In addition, two highly sensitive load cells were mounted to measure the longitudinal GRFs, and four standard parallelogram tension/compression load cells were mounted to measure the transverse GRFs. To constrain the rats to walk along the sensor module, an animal compartment constructed of Plexiglas (Fig. 3) was 
Fig. 2 Miniature force platform. Semiconductor strain gauges mounted on spring blades are sensitive to vertical and horizontal deformation of the beams caused by forces exerted when the animal moves over the wooden cover (adapted from Full and Tu 1990)

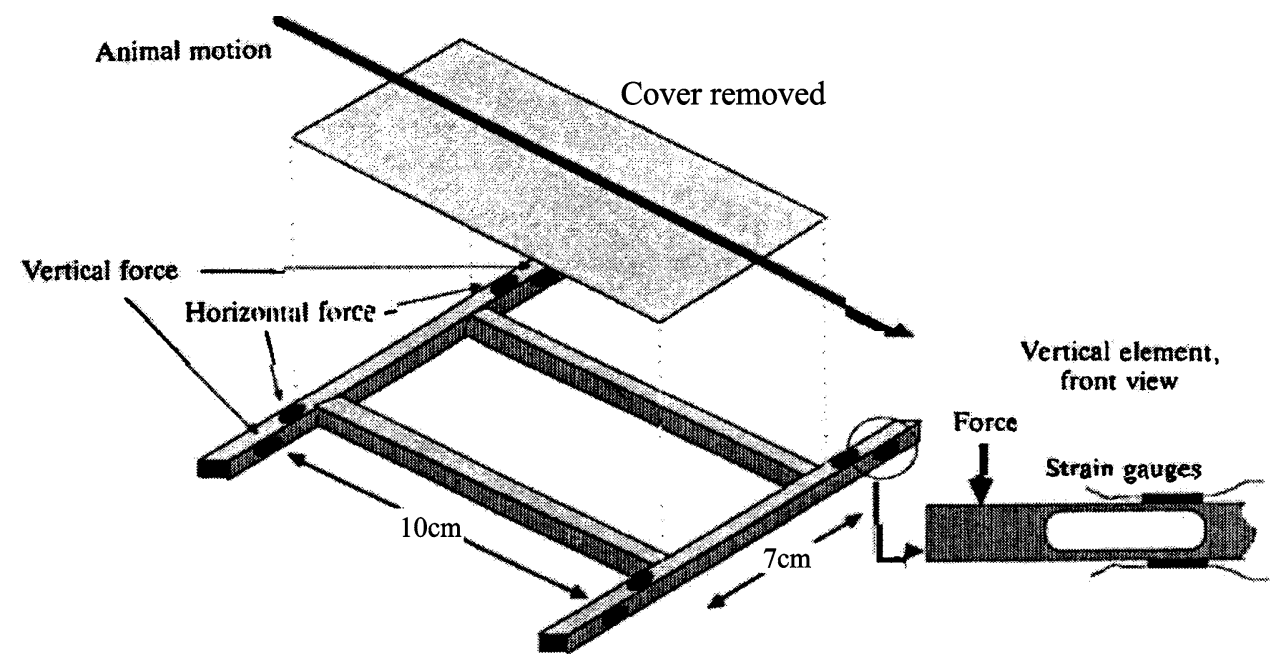

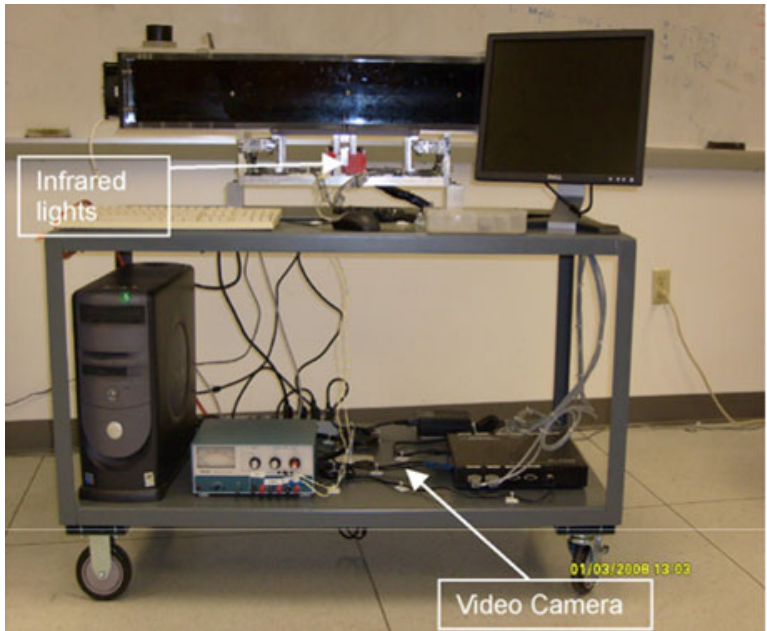

Fig. 3 A gait analysis system for rodents. The system measures locomotion parameters in four domains: force, space, time, and frequency. The measured forces are in vertical, longitudinal, and transverse directions. A video camera records bottom images of the

attached to the entrance and exit floors. Finally, a digital video camera was mounted on the base shelf to record the bottom view of the walking rats. The load cells in the sensor module were electronically connected to a carrier that provided signal conditioning and excitation power to the load cells. A LabView ${ }^{\circledR}$ program running on a PC was used for the data acquisition. Rats were encouraged to walk through the system by placing food at the exit platform. The locomotion parameters measured by the system and their definitions are listed in Table 1.

The locomotion systems above are all lab prototypes. There are also some sensor platform locomotion systems commercially available. Compared to the commercial instruments, lab prototypes have some limitations since these prototypes are still under development. The limitations

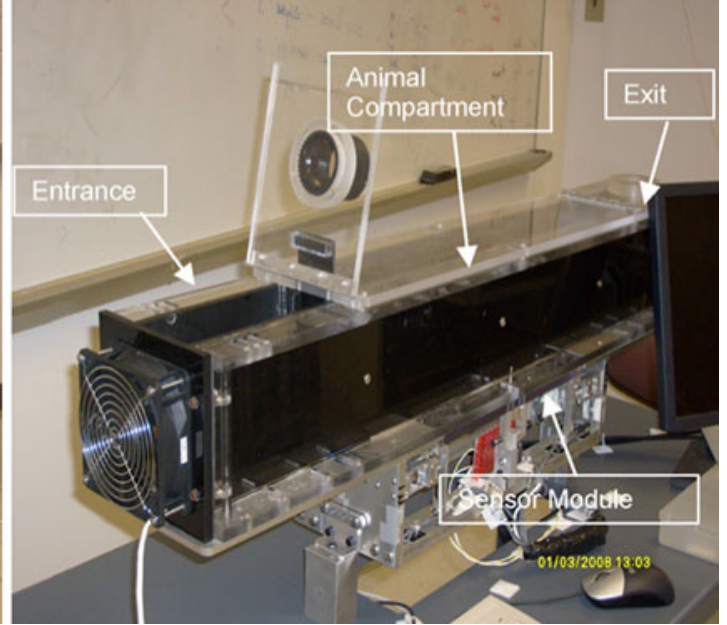

rat that walks through the system, and through synchronization of the video images and load cell signals, one can recognize the GRFs of each individual limb (adapted from Tang et al. 2009b)

may include their lower durability, lower mobility, and higher manufacturing cost.

Three popular commercial locomotion systems are DigiGait $^{\mathrm{TM}}$, CatWalk ${ }^{\mathrm{TM}}$ for animal models and GAITRite ${ }^{\circledR}$ for human beings. A digital imaging system called DigiGait $^{\mathrm{TM}}$ was developed by Mouse Specifics, Inc. (Hampton et al. 2004; Wooley et al. 2005; Li et al. 2005). When using DigiGait $^{\mathrm{TM}}$, a mouse or a rat is put on a transparent treadmill enclosed in a compartment. The treadmill belt maximizes the efficiency of the camera and image-processing software to identify paw contact with the treadmill surface at various walking and running speeds.

Similarly, Noldus Information Technology has developed a gait analysis system called CatWalk ${ }^{\mathrm{TM}}$ (Noldus IT Noldus 2006). CatWalk ${ }^{\mathrm{TM}}$ is also a video-based system 
Table 1 Description of gait parameters that were used in the locomotion analysis system developed by Tang et al. (2009b)

\begin{tabular}{|c|c|c|}
\hline & Variable & Definition \\
\hline 1. & $\mathrm{Fz}_{\max }$ & Maximum value of the vertical GRF component of a selected limb \\
\hline 2. & Stance Time & Time duration that a selected limb is in contact with the floor \\
\hline 3. & $T \_\mathrm{Fz}_{\max }$ & Time of $\mathrm{Fz}_{\max }$ normalized by the Stance Time of a selected limb \\
\hline 4. & $\mathrm{Fz}_{\text {mean }}$ & The mean value of the vertical GRF component of a selected limb $\left[\frac{\int_{\text {s.Time }} \text { Fz-d } t}{\text { Stance Time }}\right]$ \\
\hline 5. & Fzw & The Fourier transform of Fz summed over the first $50 \mathrm{~Hz}$ for a selected limb $\left[\int_{50} \mathrm{Fz} \cdot \mathrm{d} \omega\right]$ \\
\hline 6. & Stride & $\begin{array}{l}\text { Stride length of a selected limb calculated as the difference between two consecutive contact } \\
\text { positions; the contact positions are normalized by the floor length, which is } 13.25 \text { inch }\end{array}$ \\
\hline 7. & Fy $y_{\max }$ & Maximum value of the longitudinal GRF component of a selected paw \\
\hline 8. & $T_{-} \mathrm{Fy}_{\max }$ & Time of Fy $y_{\max }$ divided by Stance Time \\
\hline 9. & $F y_{\min }$ & Minimum value of the longitudinal GRF component of a selected paw \\
\hline 10. & $T \_F_{\min }$ & Time of Fymin divided by Stance Time \\
\hline 11. & Fy $y_{\text {mean }}$ & The average value of the longitudinal GRF component of a selected paw $\left[\frac{\int_{\text {s.Time }} \text { Fy.dt }}{\text { Stance Time }}\right]$ \\
\hline 12. & Fyw & The Fourier transform of Fy summed over the first $50 \mathrm{~Hz}$ for a selected paw $\left[\int_{50} \mathrm{Fy} \cdot \mathrm{d} \omega\right]$ \\
\hline 13. & $\mathrm{Fx}_{\max }$ & Maximum value of the transverse GRF component of a selected paw \\
\hline 14. & $\mathrm{Fx}_{\min }$ & Minimum value of the transverse GRF component of a selected paw \\
\hline 15. & $\mathrm{Fx}_{\text {mean }}$ & The average value of the transverse GRF component of a selected paw $\left[\frac{\int_{\text {s.Time }} \text { Fx.dt }}{\text { Stance Time }}\right]$ \\
\hline 16. & FyP & The mean value of the propelling (positive) longitudinal force of a selected limb \\
\hline 17. & FyB & The mean value of the braking (negative) longitudinal force of a selected limb \\
\hline 18. & NP & The number of samples in which the longitudinal force is propelling (positive in value); the sampling rate is $200 \mathrm{~Hz}$ \\
\hline 19. & NB & The number of samples in which the longitudinal force is braking (negative in value); the sampling rate is $200 \mathrm{~Hz}$ \\
\hline 20. & NPB & The number of times in which the longitudinal force switches sign from braking to propelling and vice versa \\
\hline
\end{tabular}

used to assess locomotion deficits and pain-induced gait adaptations in voluntarily walking mice or rats. Imageprocessing technology is employed to generate a series of gait parameters.

GAITRite $^{\circledR}$ was developed by CIR Systems, Inc. The system provides automated means of measuring spatial and temporal parameters of gait on an electronic walkway that is connected to a PC. GAITRite ${ }^{\circledR}$ focuses on measuring gait parameters of human beings. The walkway contains six sensor pads encapsulated in a roll-up carpet to produce an active area. In this arrangement, the active area is a grid, 48 sensors by 288 sensors placed on 0.5 inch centers, totaling 13,824 sensors. The walkway is portable, can be laid over any flat surface, requires minimal setup and test time, and requires no placement of any devices on the patient (van Uden and Besser 2004).

A summary of gait parameters that were used to evaluate changes in gait by DigiGait ${ }^{\mathrm{TM}}$, CatWalk ${ }^{\mathrm{TM}}$, and GAITRite $^{\circledR}$ is shown in Table 2. According to the table, it is obvious that DigiGait ${ }^{\mathrm{TM}}$, CatWalk $^{\mathrm{TM}}$, and GAITRite ${ }^{\circledR}$ can only measure gait parameters that are in time and spatial domains. Due to this limitation, some gait abnormalities which affect the GRFs may not be able to be detected by either DigiGait $^{\mathrm{TM}}$, CatWalk ${ }^{\mathrm{TM}}$, or GAITRite ${ }^{\circledR}$ (Guillot et al. 2008).

In addition, DigiGait ${ }^{\mathrm{TM}}$, CatWalk $^{\mathrm{TM}}$ and GAITRite ${ }^{\circledR}$ only provide a series of gait parameters to the users (Bozkurt et al. 2008). How the parameters are utilized depends on the users. Nevertheless, most users wish to know whether a tested animal or human being has a certain disease or not and do not desire to examine lots of gait parameters. Neither DigiGait $^{\mathrm{TM}}$, CatWalk ${ }^{\mathrm{TM}}$, nor GAITRite ${ }^{\circledR}$ can provide such diagnosis. Guillot et al. (2008) reported that a computerized gait analysis system, i.e., DigiGait ${ }^{\mathrm{TM}}$, was unable to consistently identify motor problems in 1-methyl4-phenyl-1,2,3,6-tetrahydropyridine (MPTP-treated) mice, despite a $90 \%$ deficit of striatal dopamine content. In the same report, the DigiGait ${ }^{\mathrm{TM}}$ could not distinguish SOD1-G93A, a mouse model of Amyotrophic Lateral Sclerosis (ALS), from controls up to 84 days of age.

\subsection{Wearable sensors}

The instruments introduced above are all sensor platforms. These platforms measure the locomotion parameters when animal models or human subjects are walking on them. 
Table 2 Summary of gait parameters that were used to evaluate changes in gait by DigiGait ${ }^{\mathrm{TM}}$, CatWalk $^{\mathrm{TM}}$, and GAITRite ${ }^{\circledR}$ (Berryman et al. 2009; Neumann et al. 2009; Deumens et al. 2007; van Uden and Besser 2004)

\begin{tabular}{|c|c|c|c|c|}
\hline Gait indices & Description & DigiGait $^{\mathrm{TM}}$ & CatWalk $^{\mathrm{TM}}$ & GAITRite $^{\circledR}$ \\
\hline 1. Swing time (s) & $\begin{array}{l}\text { The forward portion of the stride during which the foot is not in } \\
\text { contact with the floor }\end{array}$ & $\sqrt{ }$ & $\sqrt{ }$ & $\sqrt{ }$ \\
\hline 2. Stance/Swing (ratio) & The ratio of stance time to swing time & $\sqrt{ }$ & & \\
\hline 3. Braking Time (s) & $\begin{array}{l}\text { The time between initial foot contact with the floor to the maximal } \\
\text { foot contact }\end{array}$ & $\sqrt{ }$ & & \\
\hline 4. Stance Time (s) & $\begin{array}{l}\text { The portion of the stride in which the foot remains in contact with } \\
\text { the floor }\end{array}$ & $\sqrt{ }$ & $\sqrt{ }$ & $\sqrt{ }$ \\
\hline 5. Stance/Stride & $\begin{array}{l}\text { The percent of time that the stance time contributes to one complete } \\
\text { stride cycle }\end{array}$ & $\sqrt{ }$ & & \\
\hline 6. Stride Length $(\mathrm{cm})$ & $\begin{array}{l}\text { The distance between initial contacts of the same foot in one } \\
\text { complete stride }\end{array}$ & $\sqrt{ }$ & $\sqrt{ }$ & $\sqrt{ }$ \\
\hline 7. Stride Time (s) & The amount of time to complete one complete stride for one limb & $\sqrt{ }$ & & $\sqrt{ }$ \\
\hline 8. Swing/stride & $\begin{array}{l}\text { The percent of time that the swing time contributes to one complete } \\
\text { stride cycle }\end{array}$ & $\sqrt{ }$ & & \\
\hline 9. Propulsion/stride & $\begin{array}{l}\text { The percent of time that the propulsion time contributes to one } \\
\text { complete stride cycle }\end{array}$ & $\sqrt{ }$ & & \\
\hline 10. Maximum area $\left(\mathrm{cm}^{2}\right)$ & $\begin{array}{l}\text { The maximal foot area in contact with the floor during the stance } \\
\text { phase of the step cycle }\end{array}$ & $\sqrt{ }$ & $\sqrt{ }$ & \\
\hline 11. Stance width $(\mathrm{cm})$ & $\begin{array}{l}\text { The distance between the two front feet or the two hind feet as } \\
\text { measured from the middle of the feet area }\end{array}$ & $\sqrt{ }$ & & \\
\hline $\begin{array}{l}\text { 12. Base-of-support (BOS) } \\
(\mathrm{cm})\end{array}$ & $\begin{array}{l}\text { Distance between two feet, as measured perpendicular to the waking } \\
\text { direction }\end{array}$ & & $\sqrt{ }$ & $\sqrt{ }$ \\
\hline 13. Box length $(\mathrm{cm})$ & $\begin{array}{l}\text { Length of the box which is artificially placed around the paw prints } \\
\text { by the CatWalk }{ }^{\mathrm{TM}} \text { software }\end{array}$ & & $\sqrt{ }$ & \\
\hline 14. Box width $(\mathrm{cm})$ & $\begin{array}{l}\text { Width of the box which is artificially placed around the paw prints } \\
\text { by the CatWalk }{ }^{\mathrm{TM}} \text { software }\end{array}$ & & $\sqrt{ }$ & \\
\hline 15. Print area $\left(\mathrm{cm}^{2}\right)$ & $\begin{array}{l}\text { Total surface area of the floor contacted by the hindpaw during the } \\
\text { complete stance duration }\end{array}$ & & $\sqrt{ }$ & \\
\hline 16. Mean intensity & Mean intensity of the pixels forming the maximum area & & $\sqrt{ }$ & \\
\hline 17. Regularity index (RI) & $\begin{array}{l}\text { An index for the degree of interlimb coordination during gait, as } \\
\text { measured by the number of normal step sequence patterns (NSSP). } \\
\text { Multiplied by four (number of paws), divided by the number of } \\
\text { paw placements, and multiplied by } 100 \% \text {; RI }=(\text { NSSP } \times 4 \text { )/ } \\
\text { number of paw placements } \times 100 \% \text {, with respect to RI six NSSP } \\
\text { have been previously described (Cheng et al. 1997) and involve } \\
\text { cruciate, alternate, and rotary step patterns }\end{array}$ & & $\sqrt{ }$ & \\
\hline 18. Phase lags & $\begin{array}{l}\text { Parameters that appreciate the timing relationships between paw } \\
\text { placements. The time of initial contact of one paw (the target) is } \\
\text { related (expressed as a percentage) to the stride length of another } \\
\text { paw (the anchor). Phase lags can be calculated between the paws } \\
\text { of the same girdle (forepaws or hindpaws), between paws on the } \\
\text { same side (ipsilateral left or ipsilateral right), and between } \\
\text { diagonal paws (opposite forepaw/hindpaw) }\end{array}$ & & $\sqrt{ }$ & \\
\hline 19. Walking speed $(\mathrm{cm} / \mathrm{s})$ & The ratio of the walking distance and the corresponding time used & & & $\sqrt{ }$ \\
\hline 20. Step length $(\mathrm{cm})$ & $\begin{array}{l}\text { Distance between the placement of a foot and the subsequent } \\
\text { placement of the following foot }\end{array}$ & & & $\sqrt{ }$ \\
\hline 21. Step time (s) & Time of one single step & & & $\sqrt{ }$ \\
\hline 22. Single support time (s) & Time that the body is supported by one foot & & & $\sqrt{ }$ \\
\hline 23. Double support time (s) & Time that the body is supported by both feet & & & $\sqrt{ }$ \\
\hline 24. Toe in toe out $\left(^{\circ}\right)$ & $\begin{array}{l}\text { The angle that each foot makes with the walking direction, toe in is } \\
\text { the front of the foot pointing in towards the centerline of the } \\
\text { human body and toe out is the front of the foot pointing away from } \\
\text { the centerline of the body }\end{array}$ & & & $\sqrt{ }$ \\
\hline
\end{tabular}

The checked parameters in the column of each instrument are the ones it measures 


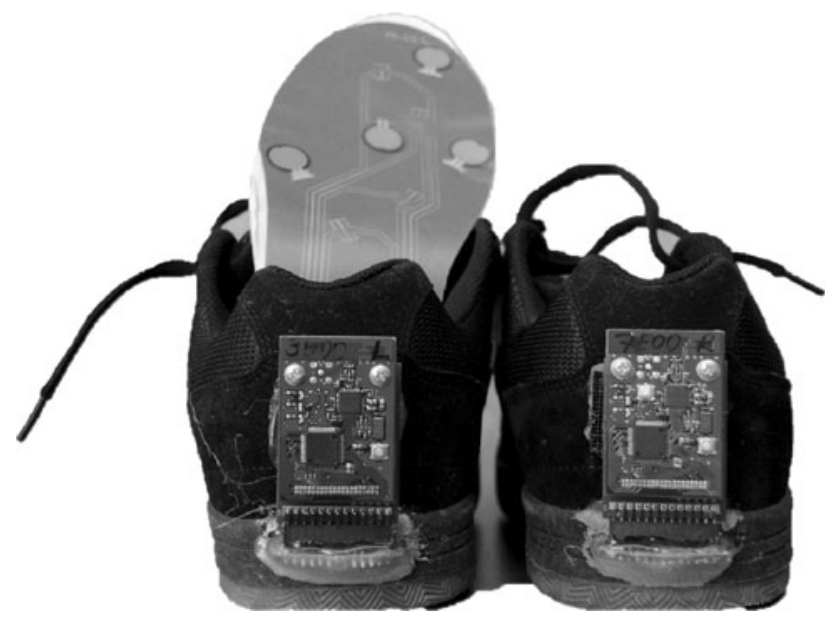

Fig. 4 SmartShoe wearable sensor system. Force-sensitive resistor (FSR) sensors located on a flexible PCB and wireless circuit and an accelerometer located at the back of the shoe (picture retaken from Lopez-Meyer et al. 2011)

They are portable but unwieldy. They are more suitable to be used in labs but irrelevant for wide-spread application. In contrast, some wearable sensor systems have been developed and are available to perform locomotion analysis as well. Compared to sensor platform systems, the wearable sensor instruments can collect locomotion data without disturbing the daily activity of the subjects. The disadvantage is that it is hard to make the sensors comfortable to be worn. Wearable sensors mainly focus on the human subjects, while the sensor platforms are usually used for animal research.

Sazonov et al. (2011) developed a shoe-based wearable sensor system, as shown in Fig. 4. The sensor system included ten pressure sensors in the two insoles, five on each one, and an accelerometer on the heel of each shoe. The accelerometer could measure the acceleration in three dimensions: vertical, longitudinal, and transverse. Data collected from the sensors could be transferred to a smartphone through wireless communication. A series of analysis programs were embedded in the smartphone that enable it to provide an analysis report to the users or doctors based on raw sensor data or statistical features. SmartShoe (Fig. 4) has been applied to the accurate prediction of energy expenditure (Sazonova et al. 2011a), monitoring of posture allocations and activities in healthy subjects (Sazonov et al. 2011), automatic detection of temporal gait parameters in post-stroke individuals (Lopez-Meyer et al. 2011), and prediction of body weight (Sazonova et al. 2011b).

Another wearable locomotion sensor system was developed by Liu et al. (2009b) as shown in Fig. 5. Three gyroscopes were used to measure angular velocities of leg segments in different positions, i.e., foot, shank, and thigh. In local coordinates of three segments, the $y$-axis was along

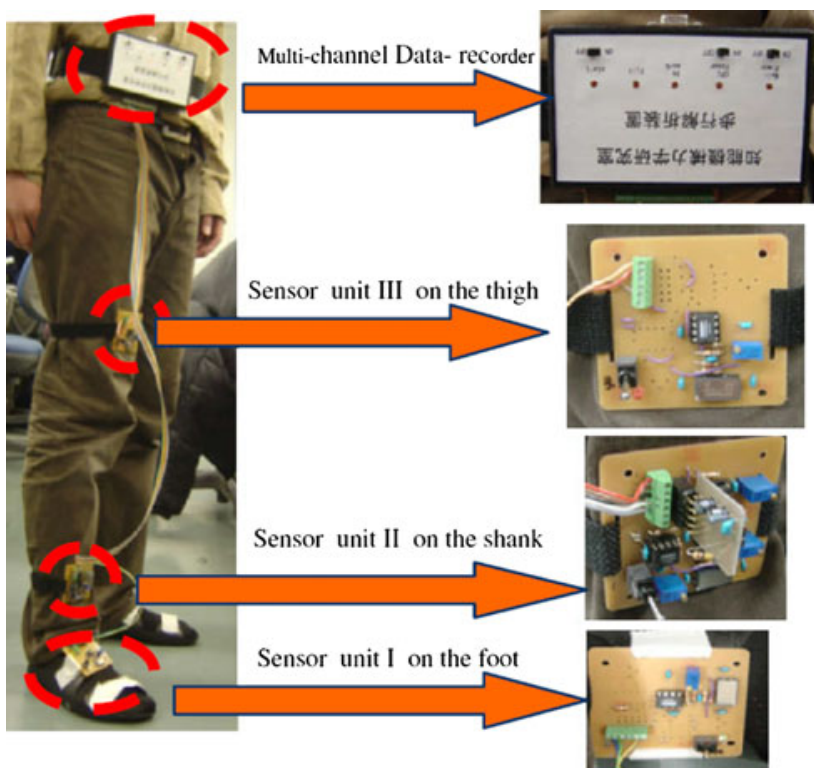

Fig. 5 Hardware system of the marker sensor system. A strap system is designed for the blinding between the sensor units and human body. The sensor unit is attached to the strap. During walking, the strap is tied around the limb to secure the position of the sensor unit (adapted from Liu et al. 2009b)

the sensing axis of the gyroscopes, and the $z$-axis was along leg-bone. The sensing axis was vertical to the medial lateral plane. A two-axis accelerometer was attached on the side of the shank to measure the accelerations along the tangent direction of the $x$-axis and the sagittal direction of the $z$-axis. In this system, the data from the accelerometer was fused with data collected from gyroscopes for cycle system calibration through supplying initial angular displacement of the attached leg segment.

Figure 6 shows a wearable locomotion analysis system called GaitShoe (Bamberg et al. 2008). Two types of data, timing parameters and pressure distribution, were measured. Force-sensitive resistors (FSRs) and polyvinylidene fluoride (PVDF) strips were placed under the foot. Two sizes of FSRs were used: FSR-402 (5 mm in diameter) and FSR-400 (12.7 $\mathrm{mm}$ in diameter). Two FSR-402 were placed underneath the heel pad, one medially and the other laterally, and two FSR-400 were placed under the first and fifth metatarsal heads. The PVDF strips were piezoelectric sensors and were configured to provide an output corresponding to dynamic pressure. Two PVDF strips were placed under the heel and the big toe.

Some wearable gait analysis instruments for human subjects are also commercially available. Xsens has a product called Xsens ForceShoe ${ }^{\mathrm{TM}}$ (http://www.Xsens.com/en/ general/forceshoe). The ForceShoe ${ }^{\mathrm{TM}}$ enables full kinematic and kinetic measurements in ambulatory settings, making it possible to analyze joint forces and torques even outside laboratory settings, e.g., in a factory or on the sports 

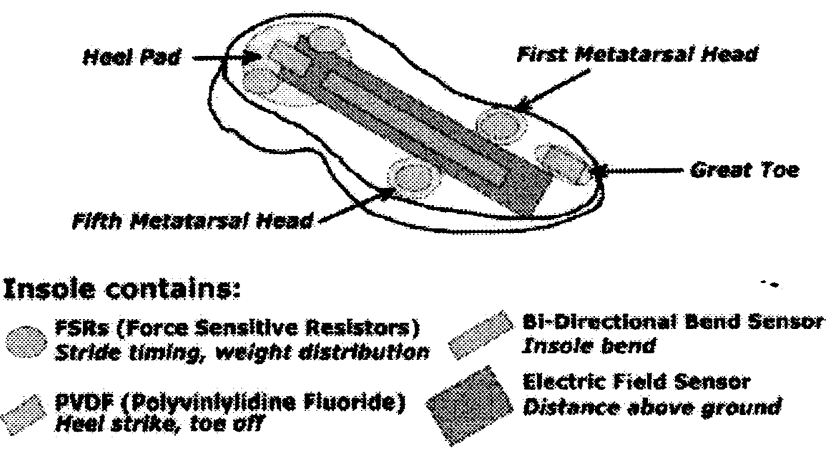
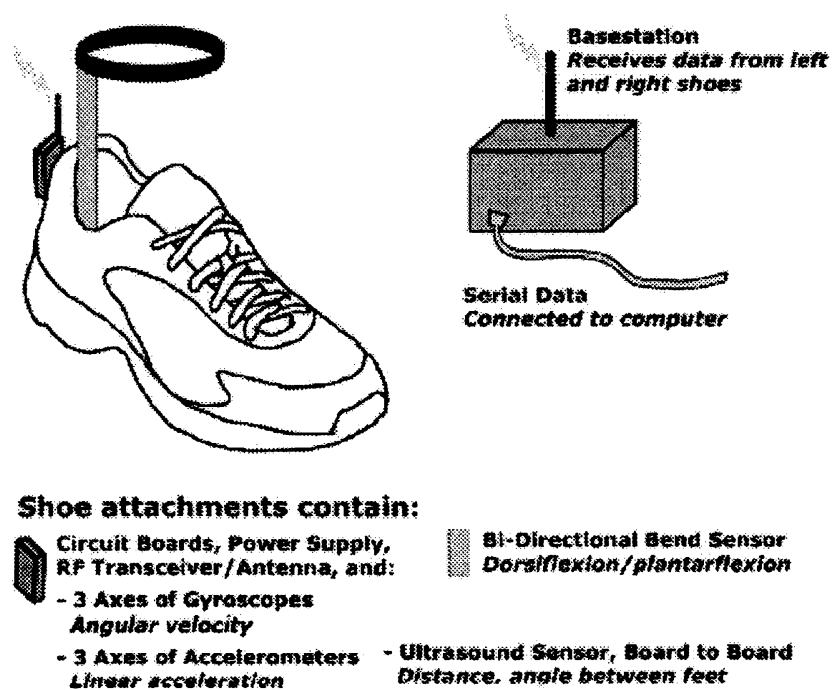

Fig. 6 Schematic of the GaitShoe system (adapted from Bamberg et al. 2008)

field. The ForceShoe ${ }^{\mathrm{TM}}$ is a unique and fully ambulatory system for 3D measurement of forces and torques under the foot, as well as 3D kinematics of the foot. The complete measurement system includes Xsens motion trackers and 6-degree-of-freedom force sensors built into an advanced lightweight carbon-fiber shoe sole which allows complete freedom of movement in and outside of the lab. Data from the ForceShoe ${ }^{\mathrm{TM}}$ is collected with time synchronized and then wirelessly transmitted to the PC using the Xsens Xbus Master (http://www.xsens.com/en/general/forceshoe). All measurement data are available in real-time to easily apply an analysis software named MT SDK. The ForceShoe ${ }^{\mathrm{TM}}$ is independent of external devices, making it ideal for research and clinical studies. It incorporates many successive strides that are difficult to re-create in a lab environment.

Charnwood Dynamic Ltd. developed a motion analysis instrument named as Codamotion cx1 unit (http://www. codamotion.com), known as CODA. CODA can do multiple motion analysis including gait analysis. CODA is the gold standard for real-time 3D movement measurement. It is portable and only weighs $5 \mathrm{~kg}$. Each CODA unit makes its own 3D measurement without reference to other cameras or units. Thus, there is no need for calibration by the user. The hardware of CODA gait analysis includes a sensor box with an extremely wide viewing angle sensor window and Gait Wand Set (GWS) as shown in Fig. 7. The CODA GWS helps to provide fast, accurate measurements of human gait. Each GWS includes a pelvic frame, thigh wands, shank wands, an ankle alignment jig, and the required markers and marker drive boxes for feet and knees. Each component can be charged and/or reprogrammed to alternative marker ID numbers to suit the user's customized marker protocol through an integral mini USB port (with USB lead supplied). GWSs come in either adult or child sizes.

\subsection{Summary}

Locomotion measurements can be classified to kinetic and kinematic measurements. In addition, different locomotion instruments have different experimental subjects, i.e., animal models and human subjects. Thus, the locomotion analysis instruments listed above can be classified as in Table 3, according to their corresponding measurement approaches and subjects. Among the gait instruments introduced above, the systems developed by Full and Tu (1990) and GaitShoe et al. (2008) are kinetic measurements. On the other hand, DigiGait $^{\mathrm{TM}}$, CatWalk ${ }^{\mathrm{TM}}$, GAITRite ${ }^{\circledR}$, CODA gait instruments, and the system developed by Liu et al. (2009b) are kinematic measurements. The gait systems developed by Clarke and Still (2001), Tang et al. (2009b), Smartshoe et al. (2011), and ForceShoe ${ }^{\mathrm{TM}}$ are for both kinetic and kinematic measurements. The instruments that focus on animal models are DigiGait ${ }^{\mathrm{TM}}$, CatWalk ${ }^{\mathrm{TM}}$, and the systems developed by Full and Tu (1990), Clarke and Still (2001), and Tang et al. (2009b). Comparably, GaitShoe, ForceShoe ${ }^{\mathrm{TM}}$, SmartShoe, GAITRite ${ }^{\circledR}$, CODA, and the system developed by Liu et al. (2009b) focus on human beings.

\section{The applications of locomotion analysis in detecting neurological disorders}

Locomotion analysis has vital applications. In this review paper, we chose to focus on its applications in detecting neurological diseases. Recently, an increasing number of people affected by neurodegenerative diseases, such as ALS, Huntington's disease (HD), and Parkinson's disease (PD), create a growing need for effective diagnosis and treatment protocols (Liao et al. 2008). As estimated, 
Fig. 7 CODA gait analysis system with a sensor box (a), a gait wand set (b) and a marker locations graph (c) (adapted from http://www. codamotion.com)

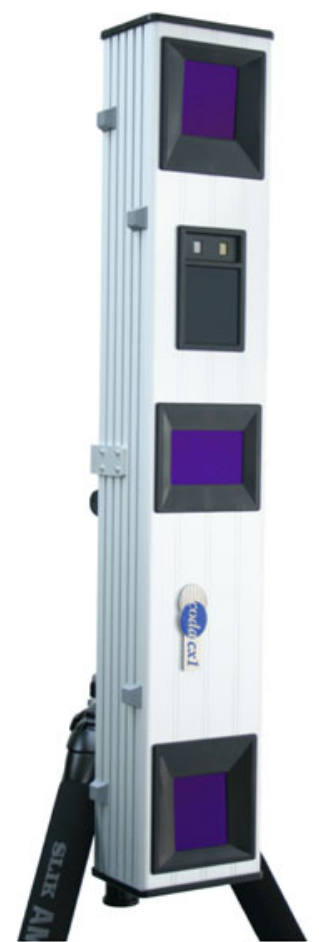

(a)

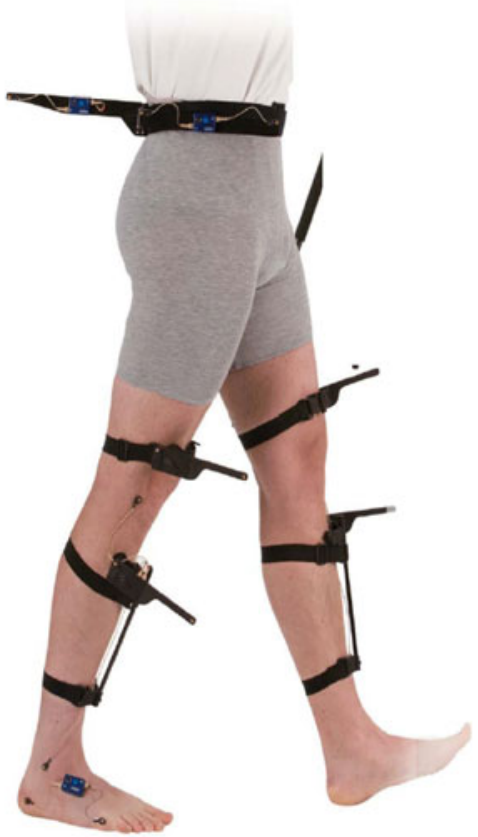

(b)

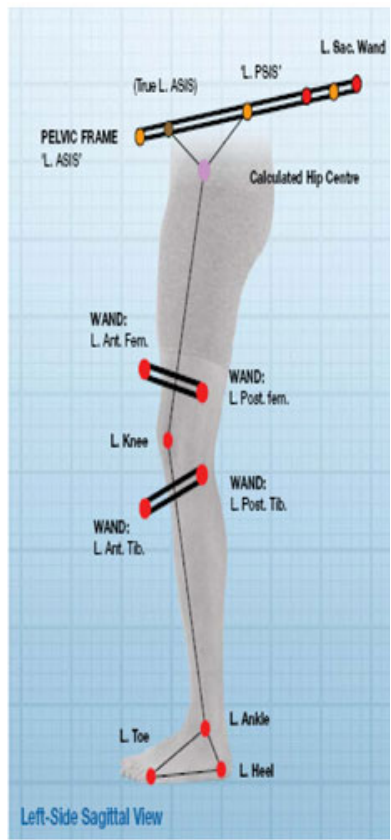

(c)
6.8 million people worldwide die every year as a result of neurological disorders, and 127 million Europeans currently suffer from one or more neurological disorders. A study estimated the total cost of multiple sclerosis in 2004 in the US was 8.3 billion dollars. In Europe, the economic cost of direct medical expenditures for neurological diseases alone was estimated at about 135 billion Euros in 2004 (Andlin-Sobocki et al. 2005). Currently, detecting the presence of neurological diseases non-invasively at an early stage is extremely difficult. In some of these diseases, locomotion analysis is the only choice for the detection, since the discovery and interpretation of minor gait abnormalities can aid in early diagnosis of the diseases (Tien et al. 2010).

For example, ALS, also called Lou Gehrig's disease, is a deadly neurodegenerative disease that affects the vast majority of a patient's motor neurons (Galan et al. 2007). ALS is difficult to diagnose at an early stage, because the symptoms are similar to those of other, often treatable, neuromuscular disorders where neurodegeneration does not occur. The diagnosis of ALS is usually based on a complete neurological examination and various clinical tests. The tests focus on the denervation and reinnervation changes in muscles and the loss of motor neurons in the ALS patients (Fischer et al. 2004). However, those tests cannot achieve early and reliable detection. Furthermore, the search for new therapeutic procedures has been hindered by the lack of early and reliable diagnosis. Early detection of ALS is
Table 3 The classification of the locomotion analysis instruments by kinetic, kinematic measurements and experimental subjects

\begin{tabular}{|c|c|c|}
\hline & Kinetic & Kinematic \\
\hline \multirow{2}{*}{ Animal models } & Full and Tu (1990) & $\begin{array}{l}\text { DigiGait }^{\mathrm{TM}} \\
\text { CatWalk }^{\mathrm{TM}}\end{array}$ \\
\hline & \multicolumn{2}{|c|}{ Tang et al. (2009b) Clarke and Still (2001) } \\
\hline \multirow{2}{*}{ Human subjects } & GaitShoe & $\begin{array}{l}\text { GAITRite }^{\circledR} \\
\text { CODA gait instruments } \\
\text { Liu et al. }(2009 \mathrm{~b})\end{array}$ \\
\hline & \multicolumn{2}{|c|}{ ForceShoe $^{\mathrm{TM}}$, SmartShoe } \\
\hline
\end{tabular}

essential not only for identifying appropriate candidate animals for inclusion in the treatment group but also for monitoring the progress and the efficacy of the treatment. Thus, early detection and the ability to assess the progression of the disease are critical for the development of new and effective therapeutic treatments for ALS (Babu et al. 2008). In this situation, locomotion analysis might be the only choice for early detection of ALS.

The locomotion analysis approach developed by Tang et al. (2009b) has been applied in three preliminary experiments on rat models for detecting neurological disorders. The results have shown that the methodology could detect early pre-symptomatic changes of ALS (a genetic 

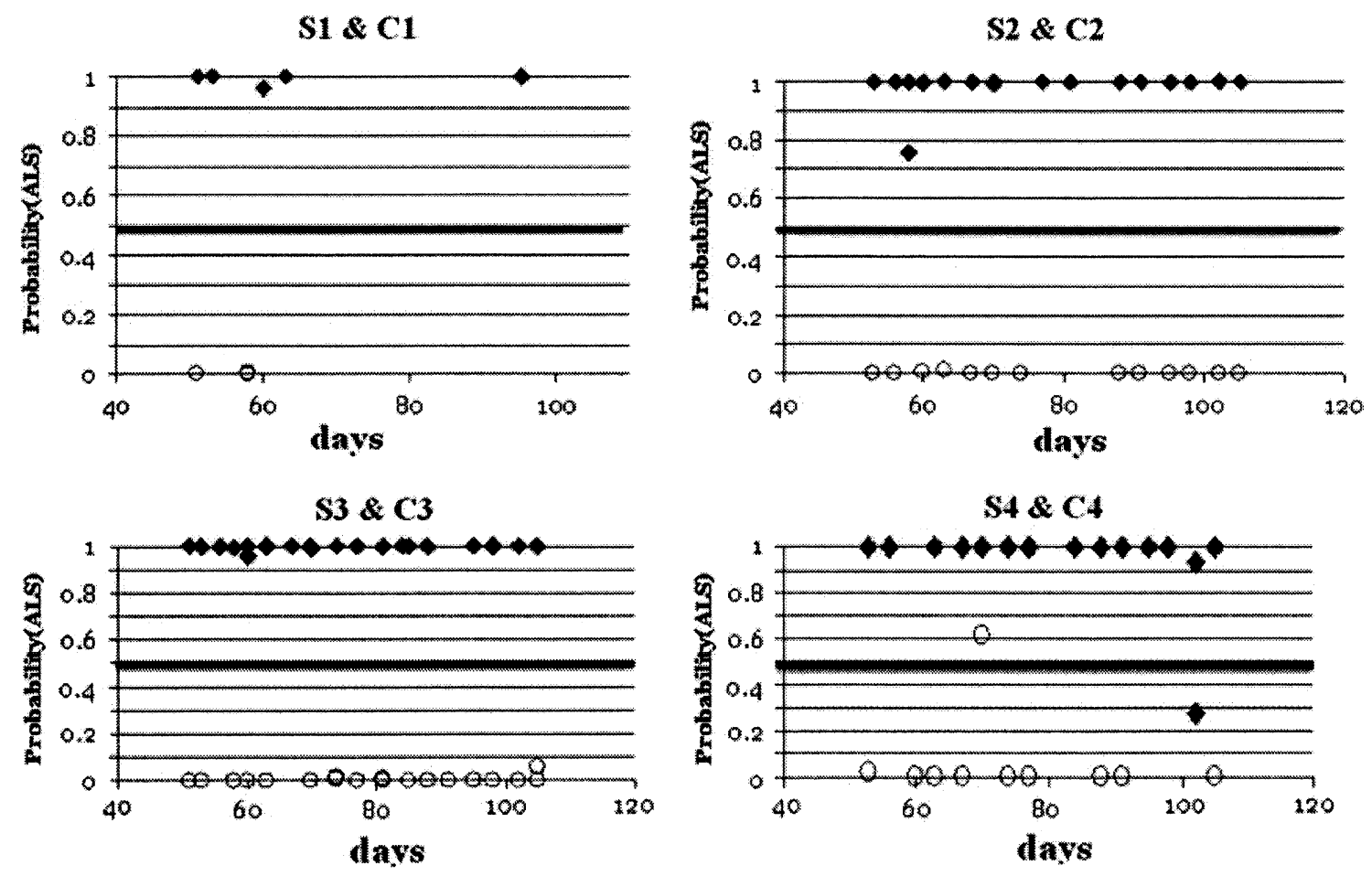

- SOD1-G93A o Control

Fig. 8 Probability of ALS for four SOD1 (S1-S4) and four control $(C 1-C 4)$ rats versus days of the animals from their births. Each data point is an average of at least two predictions. Note that probability

disease), the recovery procedure of the neuromuscular injury (physical injury), and the difference between 6-hydroxydopamine (6-OHDA) model (environmental effect) and control rats (Tang et al. 2009a, b, 2010). For the early detection of ALS, they succeed in detecting the presence of ALS when the rat models were only 51 days old (as shown in Fig. 8). This was a vast improvement over previous ALS studies, which detected the disease at 70 days old (Dunlop et al. 2003). All the detection models achieved specificity and sensitivity values of at least $90 \%$. This study could significantly impact the early diagnosis of diseases that affect the locomotion. This new methodology also had the potential to be instrumental in finding and validating new therapeutic procedures for the studied ailments.

Another gait analysis research on neurological disorders was performed by Muir and Whishaw (1999b). The HemiParkinsonian rats had been examined on the platform shown in Fig. 2. The purpose of Muir and Wishaw's work was to investigate the locomotor compensations made by Hemi-Parkinsonian rats by measuring the forces exerted by the limbs on the ground during a trotting. The impaired hindlimb was found to provide significant propulsive force and a relatively large laterally directed force. The center of the weight of Hemi-Parkinsonian rats could not follow a
(ALS) for SOD1 and control rats is close to 1.0 and 0.0, respectively, in all days except for a single day for rat \#4 (adapted from Tang et al. 2012)

sine wave, whereas the center of weight of control rats followed it at a walk, as shown in Fig. 9. The sum of the vertical GRFs from the diagonal legs had only one peak for the control group and more for the Hemi-Parkinsonian group (see Fig. 10). Yet, the methods could not detect PD early. These results demonstrated that the method was useful in the analysis of Hemi-Parkinsonian gait and provided insights to how rats could use an impaired limb to produce weight support and propulsion.

DigiGait ${ }^{\mathrm{TM}}$ has been widely employed in the research of PD, ALS (Hampton and Amende 2010), quantitative neurological traits of Down syndrome (Hampton et al. 2004), and ethanol's effects on gait dynamics (Kale et al. 2004), in mice models. Moreover, DigiGait ${ }^{\mathrm{TM}}$ was also used to study muscular dystrophy on the delta-sarcoglycan-deficient hamster models (Hampton et al. 2011). The shortcoming of DigiGait $^{\mathrm{TM}}$ is the locomotion parameters (as shown in Table 2) are delivered to the users, neither mathematical models nor detection results can be provided by the system.

The locomotion analysis is also broadly used on human subjects. Liu et al. (2009b) used the wearable sensor system as shown in Fig. 5 for estimating muscular tensions instead of electromyography (EMG). Analysis of human walking pattern by the eight gait phases more directly 
(a)
Control

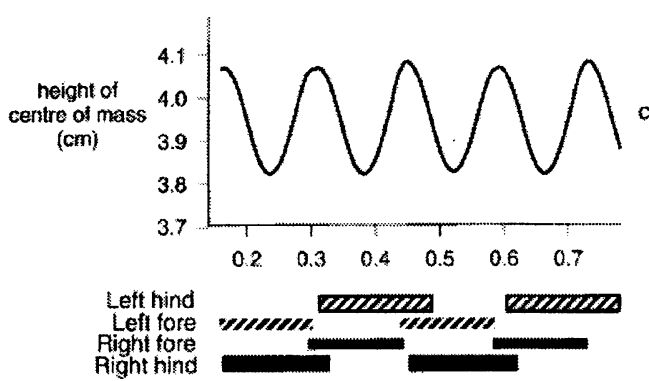

Fig. 9 Height of the body center of mass plotted against time for control animals $(\mathbf{a} ; n=6)$ and striatal dopaminergic (DA)-depleted animals $(\mathbf{b} ; n=5)$. Solid and hatched bars demonstrate the duration of ground contact for each limb. Note that the center of mass (b)

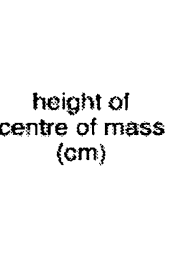

DA depleted

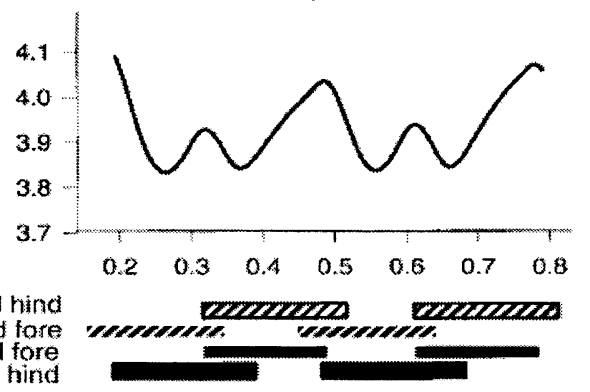

oscillates evenly twice during each stride in control animals, but oscillates unevenly during each stride in animals with DA depletion (adapted from Muir and Whishaw 1999b)

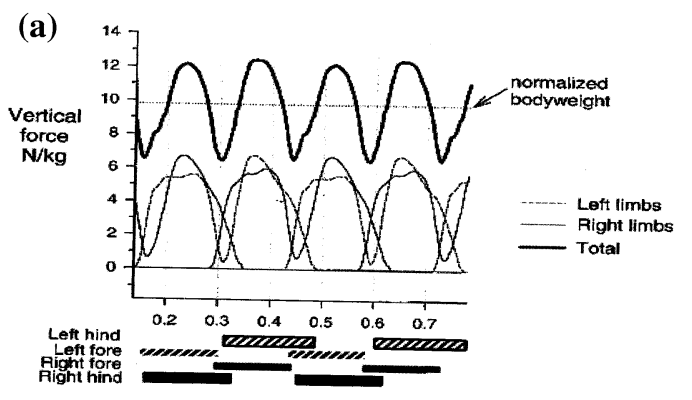

Fig. 10 Summed GRFs in the vertical direction for control (a) and DA depleted animal model (b). Notice the two peaks in vertical force produced during contact of the good hindlimb and bad forelimb (asterisk in b) (adapted from Muir and Whishaw 1999b)

identifies the functional significance (muscular tensions) of the different motions accruing at the individual joints and segments. For example, in the mid stance phase including the early interval and the late interval, the early interval has the body over the mid foot, in which the quadriceps and the soleus muscles are in activity; the later interval has body over the forefoot, in which the soleus and the gastrocnemius are only extensor muscles around the tibia. They combined the developed wearable human motion analysis sensor system with a reaction force sensor worn underfoot to estimate muscular tensions. This technology can be directly adapted in detecting neurological disorders, because muscular tensions are important indicators for neurological disorders (Tang et al. 2009a).

The GaitShoe (as shown in Fig. 6) was built to be worn in any shoe without interfering with gait and was designed to collect data unobtrusively, in any environment and over long periods. GaitShoe could measure the change in gait over extended time and it had been used in neurological exams to diagnose dementias and a number of neurologic disorders (Morris 2004). The calibrated sensor outputs were analyzed and validated with results obtained simultaneously from the Biomotion Laboratory at Massachusetts General Hospital. The GaitShoe was proved to be highly capable of detecting heel-strike and toe-off, as well as estimating foot orientation and position, inter alia (Bamberg et al. 2008). The preliminary results demonstrated that GaitShoe was capable of the analysis of gait in untraditional ways, such as over long-time periods and in the home environment, through the use of multisensory pattern recognition. Fast processing of the data stream could provide real-time feedback for the applications such as sports medicine, electro stimulation, or physical therapy. Additional research with the GaitShoe sensor outputs had involved the application of standard pattern-recognition techniques to discriminate between healthy gait and PD gait. The outputs could also recognize the individual gaits of various subjects. The real-time analysis of the collected data could provide therapeutic feedback to investigate interactive applications for physical therapy (Morris 2004; Paradiso et al. 2004).

\section{Discussion}

A major advantage of locomotion analysis is its non-invasive characteristics, compared to other available clinical tests. This is extremely important for some specific diseases, such as neurological diseases. In addition, locomotion analysis systems are simple to implement, carry a 
low cost, and reduce the risk of complication. Therefore, they have been widely used to detect neurological diseases (Hamers et al. 2001; Koopmans et al. 2006; Neumann et al. 2009).

Locomotion analysis instruments make the gait measurements objective. There is a definite need for objective measurement of gaits. Without it, the quality of treatment decisions is reduced due to subjective and often unreliable assessments. Objective measurements must also be employed if one is to demonstrate the efficacy of a treatment protocol (Wall and Brunt 1996).

Gait patterns can be affected by many factors, such as emotional and environmental factors. Thus, the challenge of locomotion analysis is how to prove the gait abnormalities measured are caused by the target disorders rather than other factors. In other words, the challenge is how to eliminate false positives. One solution for this is to validate the results of locomotion analysis by using other diagnostic approaches, such as image-processing-based clinical decision support (Zillner and Sonntag 2012) and genomic data integration (Zhou et al. 2012).

\section{Conclusion}

This review summarized the recent development in the field of locomotion analysis, including locomotion analysis instruments and their applications in detecting neurological disorders. The experimental subjects of locomotion analysis consisted of animal models and human beings.

Locomotion analysis, a subgenre study of motion analysis as well as an important division of behavioral research, is usually used as a non-invasive approach to clinical diagnosis. It is impossible to fully summarize locomotion study in one paper. However, this review focused on creating a rough picture of locomotion analysis in detection of neurological disorders according to the authors' experience in the field. The materials provided here is to the authors' best knowledge.

Acknowledgments The authors deeply appreciate Dr. Uri Tasch, professor of Mechanical Engineering in the University of Maryland Baltimore County, who led the authors to the field of locomotion analysis. The authors also appreciate the anonymous referees for their valuable comments. At last, the authors thank the writing consultants from the writing center of the University of Alabama.

\section{References}

Andlin-Sobocki P, Jonsson B, Wittchen HU, Olesen J (2005) Cost of disorders of the brain in Europe. Eur J Neurol 1:1-27

Babu GN, Kumar A, Chandra R, Puri SK, Singh RL, Kalita J, Misra UK (2008) Oxidant-antioxidant imbalance in the erythrocytes of sporadic amyotrophic lateral sclerosis patients correlates with the progression of disease. Neurochem Int 52(6):1284-1289. doi: 10.1016/j.neuint.2008.01.009

Bamberg SJ, Benbasat AY, Scarborough DM, Krebs DE, Paradiso JA (2008) Gait analysis using a shoe-integrated wireless sensor system. IEEE Trans Inf Technol Biomed 12(4):413-423

Berryman ER, Harris RL, Moalli M, Bagi CM (2009) Digigait ${ }^{\mathrm{TM}}$ quantitation of gait dynamics in rat rheumatoid arthritis model. J Musculoskelet Neuronal Interact 9(2):89-98

Bozkurt A, Deumens R, Scheffel J, O’Dey DM, Weis J, Joosten EA, Führmann T, Brook GA, Pallua N (2008) CatWalk gait analysis in assessment of functional recovery after sciatic nerve injury. J Neurosci Methods 173(1):91-98. doi:10.1016/j.jneumeth.2008. 05.020

Cheng H, Almstrom S, Gimenez-Llort L, Chang R, Ove Ogren S, Hoffer B, Olson L (1997) Gait analysis of adult paraplegic rats after spinal cord repair. Exp Neurol 148(2):544-557

Clarke KA, Still J (2001) Development and consistency of gait in the mouse. Physiol Behav 73(1-2):159-164. doi:10.1016/s00319384(01)00444-9

Costa ACS, Walsh K, Davisson MT (1999) Motor dysfunction in a mouse model for Down syndrome. Physiol Behav 68(1-2): 211-220. doi:10.1016/s0031-9384(99)00178-x

Deumens R, Jaken RJP, Marcus MAE, Joosten EAJ (2007) The CatWalk gait analysis in assessment of both dynamic and static gait changes after adult rat sciatic nerve resection. J Neurosci Methods 164(1):120-130. doi:10.1016/j.jneumeth.2007.04.009

Dunlop J, Beal McIlvain H, She Y, Howland DS (2003) Impaired spinal cord glutamate transport capacity and reduced sensitivity to riluzole in a transgenic superoxide dismutase mutant rat model of amyotrophic lateral sclerosis. J Neurosci 23(5):1688-1696

Fischer LR, Culver DG, Tennant P, Davis AA, Wang M, CastellanoSanchez A, Khan J, Polak MA, Glass JD (2004) Amyotrophic lateral sclerosis is a distal axonopathy: evidence in mice and man. Exp Neurol 185(2):232-240. doi:10.1016/j.expneurol.2003.10. 004

Full RJ, Tu MS (1990) Mechanics of six-legged runners. J Exp Biol 148:129-146

Galan L, Vela A, Guerrero A, Barcia JA, Garcia-Verdugo JM, Matias-Guiu J (2007) Experimental models of amyotrophic lateral sclerosis. Neurologia 22(6):381-388

Guillot TS, Asress SA, Richardson JR, Glass JD, Miller GW (2008) Treadmill gait analysis does not detect motor deficits in animal models of Parkinson's disease or amyotrophic lateral sclerosis. J Mot Behav 40(6):568-577

Hamers FP, Lankhorst AJ, van Laar TJ, Veldhuis WB, Gispen WH (2001) Automated quantitative gait analysis during overground locomotion in the rat: its application to spinal cord contusion and transection injuries. J Neurotrauma 18(2):187-201

Hampton TG, Amende I (2010) Treadmill gait analysis characterizes gait alterations in Parkinson's disease and amyotrophic lateral sclerosis mouse models. J Mot Behav 42(1):1-4

Hampton TG, Stasko MR, Kale A, Amende I, Costa ACS (2004) Gait dynamics in trisomic mice: quantitative neurological traits of Down syndrome. Physiol Behav 82(2-3):381-389. doi:10.1016/ j.physbeh.2004.04.006

Hampton TG, Kale A, Amende I, Tang W, McCue S, Bhagavan HN, VanDongen CG (2011) Gait disturbances in dystrophic hamsters. J Biomed Biotechnol 235354:13

Heglund NC (1981) A simple design for a force-plate to measure ground reaction forces. J Exp Biol 93:333-338

Kale A, Amende I, Meyer GP, Crabbe JC, Hampton TG (2004) Ethanol's effects on gait dynamics in mice investigated by ventral plane videography. Alcohol Clin Exp Res 28(12):18391848

Koopmans GC, Brans M, Gomez-Pinilla F, Duis S, Gispen WH, Torres-Aleman I, Joosten EA, Hamers FP (2006) Circulating 
insulin-like growth factor I and functional recovery from spinal cord injury under enriched housing conditions. Eur J Neurosci 23(4):1035-1046

Li S, Kim J-E, Budel S, Hampton TG, Strittmatter SM (2005) Transgenic inhibition of Nogo-66 receptor function allows axonal sprouting and improved locomotion after spinal injury. Mol Cell Neurosci 29(1):26-39. doi:10.1016/j.mcn.2004.12.008

Liao F, Wang J, He P (2008) Multi-resolution entropy analysis of gait symmetry in neurological degenerative diseases and amyotrophic lateral sclerosis. Med Eng Phys 30(3):299-310. doi:10.1016/ j.medengphy.2007.04.014

Liu J, Neerchal NK, Tasch U, Dyer RM, Rajkondawar PG (2009a) Enhancing the prediction accuracy of bovine lameness models through transformations of limb movement variables. J Dairy Sci 92(6):2539-2550. doi:10.3168/jds.2008-1301

Liu T, Inoue Y, Shibata K (2009b) Development of a wearable sensor system for quantitative gait analysis. Measurement 42(7):978988. doi:10.1016/j.measurement.2009.02.002

Lopez-Meyer P, Fulk GD, Sazonov ES (2011) Automatic detection of temporal gait parameters in poststroke individuals. IEEE Trans Inf Technol Biomed 15(4):594-601

Morris SJ (2004) A shoe-integrated sensor system for wireless gait analysis and real-time therapeutic feedback. Harvard/MIT, Cambridge

Morris RG, Lawson SEM (2009) A review and evaluation of available gait analysis technologies, and their potential for the measurement of impact transmission. Newcastle University, UK

Muir GD, Whishaw IQ (1999a) Complete locomotor recovery following corticospinal tract lesions: measurement of ground reaction forces during overground locomotion in rats. Behav Brain Res 103(1):45-53. doi:10.1016/s0166-4328(99)00018-2

Muir GD, Whishaw IQ (1999b) Ground reaction forces in locomoting hemi-parkinsonian rats: a definitive test for impairments and compensations. Exp Brain Res 126(3):307-314

Neumann M, Wang Y, Kim S, Hong SM, Jeng L, Bilgen M, Liu J (2009) Assessing gait impairment following experimental traumatic brain injury in mice. J Neurosci Methods 176(1):34-44. doi:10.1016/j.jneumeth.2008.08.026

Noldus IT (2006) CatWalk. http://www.noldus.com/animal-behaviorresearch/products/catwalk

Paradiso JA, Morris SJ, Benbasat AY, Asmussen E (2004) Interactive therapy with instrumented footwear. Paper presented at the $\mathrm{CHI}$ ' 04 extended abstracts on Human factors in computing systems, Vienna, Austria

Reiner A, Lafferty DC, Wang HB, Del Mar N, Deng YP (2012) The group 2 metabotropic glutamate receptor agonist LY379268 rescues neuronal, neurochemical and motor abnormalities in R6/2 Huntington's disease mice. Neurobiol Dis 47(1):75-91

Sazonov ES, Fulk G, Hill J, Schutz Y, Browning R (2011) Monitoring of posture allocations and activities by a shoe-based wearable sensor. IEEE Trans Biomed Eng 58(4):983-990

Sazonova NA, Browning RC, Sazonov ES (2011a) Accurate prediction of energy expenditure using a shoe-based activity monitor. Med Sci Sports Exerc 43(7):1312-1321

Sazonova NA, Browning RC, Sazonov ES (2011b) Prediction of bodyweight and energy expenditure using point pressure and foot acceleration measurements. Open Biomed Eng J 5:110-115

Suresh Babu SR, Sunandhini RL, Sridevi D, Periasamy P, Namasivayam A (2012) Locomotor behavior of bonnet monkeys after spinal contusion injury: footprint study. Synapse 66(6):509-521
Tang W, Lovering RM, Roche JA, Bloch RJ, Neerchal NK, Tasch U (2009a) Gait analysis of locomotory impairment in rats before and after neuromuscular injury. J Neurosci Methods 181(2):249_ 256. doi:10.1016/j.jneumeth.2009.04.027

Tang W, Tasch U, Neerchal NK, Zhu L, Yarowsky P (2009b) Measuring early pre-symptomatic changes in locomotion of SOD1-G93A rats-a rodent model of amyotrophic lateral sclerosis. J Neurosci Methods 176(2):254-262. doi:10.1016/j. jneumeth.2008.08.032

Tang W, McDowell K, Limsam M, Neerchal NK, Yarowsky P, Tasch U (2010) Locomotion analysis of Sprague-Dawley rats before and after injecting 6-OHDA. Behav Brain Res 210(1):131-133. doi:10.1016/j.bbr.2010.02.012

Tang W, Yarowsky P, Tasch U (2012) Detecting ALS and Parkinson's disease in rats through locomotion analysis. Netw Model Anal Health Inf Bioinf 1:63-68. doi:10.1007/s13721-0120004-8

Tasch U, Moubarak P, Tang W, Zhu L, Lovering RM, Roche JA, Bloch RJ (2008) An instrument that simultaneously measures spatial gait parameters and ground reaction forces of locomoting rats. In: The 9th biennial ASME conference on engineering systems design and analysis (ESDA 08), Haifa, Israel, July 7-9 2008

Thomsen PT, Munksgaard L, Sørensen JT (2012) Locomotion scores and lying behaviour are indicators of hoof lesions in dairy cows. Vet J 193(3):644-647

Tien I, Glaser SD, Bajcsy R, Goodin DS, Aminoff MJ (2010) Results of using a wireless inertial measuring system to quantify gait motions in control subjects. IEEE Trans Inf Technol Biomed 14(4):904-915. doi:10.1109/titb.2009.2021650

Toro B, Farren P, Nester C (2002) Review of observational gait assessment in clinical practice. Physiotherapy 88(11):701. doi: 10.1016/s0031-9406(05)60117-4

van Uden CJ, Besser MP (2004) Test-retest reliability of temporal and spatial gait characteristics measured with an instrumented walkway system (GAITRite). BMC Musculoskelet Disord 5:13

Wall JC, Brunt D (1996) Clinical gait analysis: temporal and distance parameters. In: Van Deussen JV (ed) Assesment in occupational therapy and physical therapy

Wooley CM, Sher RB, Kale A, Frankel WN, Cox GA, Seburn KL (2005) Gait analysis detects early changes in transgenic SOD1 (G93A) mice. Muscle Nerve 32:43-50

Woollacott M, Shumway-Cook A (2002) Attention and the control of posture and gait: a review of an emerging area of research. Gait Posture 16(1):1-14. doi:10.1016/s0966-6362(01)00156-4

Wu Y, Cai S, Lu M, Zhong Z, He J, Zheng F, Xu F, Shi L, Xia C (2012) A gait cadence measurement method based on match filter and turns detection for human locomotion monitoring. In: IEEE-EMBS international conference on biomedical and health informatics, Hong Kong, China, pp 530-533

Young MD, Streicher MC, Beck RJ, van den Bogert AJ, Tajaddini A, Davis BL (2012) Simulation of lower limb axial arterial length change during locomotion. J Biomech 45(8):1485-1490

Zhou Y, Qureshi R, Sacan A (2012) Data simulation and regulatory network reconstruction from time-series microarray data using stepwise multiple linear regression. Netw Model Anal Health Inf Bioinf 1(1):3-17. doi:10.1007/s13721-012-0008-4

Zillner S, Sonntag D (2012) Image metadata reasoning for improved clinical decision support. Netw Model Anal Health Inf Bioinf 1(1):37-46. doi:10.1007/s13721-012-0003-9 